\title{
Comparison of structural health assessment capabilities in epoxy - carbon black and epoxy - carbon nanotube nanocomposites
}

\author{
F. Inam $^{1 *}$, B.R. Bhat ${ }^{2}$, N. Luhyna ${ }^{3}$, T. Vo ${ }^{1}$ \\ ${ }^{1}$ Northumbria University, Faculty of Engineering and Environment, Newcastle upon Tyne, NE1 8ST, United Kingdom \\ ${ }^{2}$ National Institute of Technology Karnataka, Department of Chemistry, Catalysis and Materials Laboratory, Surathkal, \\ Srinivasanagar-575025, India \\ ${ }^{3}$ Advanced Composite Training and Development Centre (Airbus) and Glyndŵr University, Hawarden and Wrexham, \\ CH5 3US and LL11 2AW, United Kingdom
}

Received 10 July 2013; accepted in revised form 9 September 2013

\begin{abstract}
A novel method for comparing structural health of different types of brittle epoxy nanocomposites filled with carbon nanostructured fillers is presented. Epoxy -0.2 vol\% carbon black $(\mathrm{CB})$ and epoxy -0.2 vol\% carbon nanotube (CNT) nanocomposite bars were prepared by calendering and thermal curing. Nanocomposite bars were subjected to Vickers diamond indentation to produce sub-surface damage. Electrical conductivities were analysed by 4-point method to estimate the structural damage caused by indentation. For comprehensive comparison, fracture toughness and percolation threshold were analysed as well. Because of the systematically induced indentation damage, a sharp decrease of $89 \%$ was observed in the electrical conductivity of epoxy - CNT nanocomposite as compared to $25 \%$ in the electrical conductivity of epoxy - CB nanocomposite. CNTs impart superior damage sensing capability in brittle nanocomposite structures, in comparison to CB, due to their high aspect ratio (fibrous nature) and high electrical conductivity.
\end{abstract}

Keywords: nanocomposites, carbon nanotubes, epoxy, material testing, smart polymers

\section{Introduction}

Carbon nanotubes (CNTs), discovered accidentally in 1991 [1], have been widely investigated for their addition in polymer [2-5], ceramic [6] and metal [7] matrices to prepare nanocomposites owing to the combination of the superlative mechanical, thermal, and electronic properties attributed to them. CNTfilled polymer matrix composite materials are subject of significant research for their utilisation in an increasing number of industrial applications including energy, transportation, defence, automotive, aerospace, sporting goods, and infrastructure sectors [8]. Particularly among brittle epoxy materials, CNTs have been reported to significantly improve mechan- ical, thermal and electrical properties of the epoxy nanocomposites [9]. Apart from these improvements, CNTs also offer structural damage sensing ability to epoxy and the subject has been widely investigated [10-16] too. Structural health monitoring (SHM) is a type of a Non-destructive Evaluation (NDE) technique that essentially involves the strategic embedding of conductive filler into a structure to allow continuous and remote monitoring for damage, deformation and failure. SHM technology is applied increasingly for research and industrial purposes as a potential tool for quality assurance $[17,18]$. However, many of the developed and available NDE technologies are complex, expensive and require signif-

\footnotetext{
${ }^{*}$ Corresponding author, e-mail: fawad.inam@northumbria.ac.uk (C) BME-PT
} 
icant calibration with the passage of time. Nanostructured carbon embedded systems have proven to be more sensitive towards structural damage [10-16]. Most of the previous researched methods incorporating CNTs were based on the sensing of damage of long-fibre or woven reinforced epoxy [10-16] or for other brittle matrices like cement for civil structures [19]. Such studies aimed to induce damage systematically to the nanocomposite sample using tensile $[10,11]$ and bending $[11,12]$ modes. Anand and Mahapatra [13] dispersed carbon black (CB) and CNTs in epoxy thins and conducted a quasi-static and dynamic strain sensing study. However, there was no characterisation of damage in that report. Thostenson and Chou [14] studied in situ sensing to detect localized damage in CNT-filled glass fiber composites but no comparison was made with CBfilled system. Recently, Nagashpour and Hoa [15] investigated in situ monitoring of through-thickness strain in glass fiber/epoxy composite laminates using carbon nanotube sensors. Kostopoulos et al. [16] reported improved structural health monitoring capability in CNT-filled carbon fibre-reinforced epoxy system as compared to carbon fibre-reinforced epoxy. However, no comparisons were made for CB-filled systems in these studies.

A novel method of assessing structural damage in epoxy by analysing change in the electrical conductivity of epoxy-CNT nanocomposite is presented in this letter. A comparison between the sensitivity of CNT based nanocomposites with carbon black based nanocomposites has been made.

\section{Experimental}

The epoxy matrix used in this study consists of a modified DGEBA-based epoxy resin (GY250, a diglycidyl ether of bisphenol A) with a piperidine hardener, supplied by Ciba-Geigy, India. Carbon black powder $(<50 \mathrm{~nm}$, grade $633100,>99 \%$ pure $)$ was supplied by Sigma Aldrich, UK. As per supplier, carbon black powder was produced by laser technique. Helium pycnometry (AccuPyc II 1340, micrometrics, UK) was used to evaluate densities of CNTs and CB for the calculation of filler volume fraction. Density of CB powder was found to be $\sim 1 \mathrm{~g} / \mathrm{cm}^{3}$. The multiwall CNTs (grade: Elicarb) used in this study were kindly supplied by Thomas Swan, UK. They were synthesised by the Chemical Vapour Deposition (CVD) method and have an entangled cotton-like form. The CNTs have density of $\sim 1.1 \mathrm{~g} / \mathrm{cm}^{3}$, an average outer diameter of $15 \mathrm{~nm}$ and lengths of up to 50 microns. CNT dimensions were measured as per technique mentioned elsewhere [20]. For reducing the non-carbonaceous content in CNT powder, an acid treatment was performed using a mixture of nitric $\left(\mathrm{HNO}_{3}, 90 \%\right)$ and sulfuric $\left(\mathrm{H}_{2} \mathrm{SO}_{4}\right.$, $90 \%$ ) acids. Acids were supplied by Sigma-Aldrich, UK. Distilled water ( 20 vol\%) was used to dilute the acids. In order to produce pure CNTs, the asreceived CNTs $(400 \mathrm{mg}$ ) were mixed with $200 \mathrm{~mL}$ acidic solution. Both acids were equally mixed in the solution. The acid-CNT mixture was homogenised by stirring with a glass rod on heating plate $\left(\sim 85^{\circ} \mathrm{C}\right)$ for $30 \mathrm{~min}$ and then bath ultrasonicated for $2 \mathrm{~h}$. The resulting CNT dispersion was thoroughly washed with distilled water until the filtrate was colourless and neutral $(\mathrm{pH} \sim 7)$ after filtration. A Whatman filter paper of $1 \mu \mathrm{m}$ was used. The purified CNTs were then dried for $48 \mathrm{~h}$ at $100^{\circ} \mathrm{C}$ in an oven. With the aid of Thermogravimetric analysis (TGA) technique, the amount of carbonaceous content in CNT powder was measured by recording the dry mass of remnant after complete oxidation of carbonaceous content. TGA was performed using TA Instruments (UK) SDT Q600 thermo gravimetric analyser. All specimens were examined on platinum pans in the range $30-650^{\circ} \mathrm{C}$. A heating rate of $5^{\circ} \mathrm{C} /$ minute in flowing air (at $180 \mathrm{~mL} /$ minute) was used. Powder sample masses ranged from 30-40 mg. CNTs were purified to $>99 \%$ (i.e. carbonaceous content) by acid treatment. Average length of CNT was found to be $\sim 40$ microns. No change in diameter was observed after acid treatment.

For producing epoxy -0.2 vol\% CNT nanocomposites, a batch of CNTs were aggressively mixed by high-speed mixing (RPM: 2400, Silverson L5 Series, UK,) with the epoxy resin (without hardener) for 10 mins. This suspension was then added batch-wise to a mini-calender (Exakt, Germany) for final high shear mixing. The gap size between the alumina rolls of the mini-calender was $3 \mu \mathrm{m}$ and the speed was set to 250 ( $1^{\text {st }}$ roll $), 70$ ( $2^{\text {nd }}$ roll $)$ and $165 \mathrm{rpm}$ ( $3^{\text {rd }}$ roll). The dwell time of each batch of suspension was $\sim 3 \mathrm{~min}$. The suspension was collected, mixed with the hardener for $15 \mathrm{~min}$ by highspeed mixing (RPM: 2000) for 5 mins. The suspension was poured in an open teflon mould ( 5 samples) and then cured for $16 \mathrm{~h}$ at $120^{\circ} \mathrm{C}$. The same proce- 
dure was used for producing 5 bars of epoxy 0.2 vol\% CB nanocomposites and a bar of monolithic epoxy for comparison.

All samples were produced on the same day and were placed in a desiccator. After 15 hours they were removed from desiccator and all sample surfaces were cleaned by gentle wiping using a lint-free cloth soaked with isopropanol. After silver coating (Figure 1), they were then immediately indented and electrical conductivities were measured (at least 5 observations were made). The Vickers indentations were performed using a Zwick microhardness tester (500 g load with 10 seconds of indentation time). $\mathrm{HV} \mu$ micro Vickers hardness tester was supplied by Zwick (UK). Five indents (equally spaced) were produced on each bar and the bulk electrical conductivity of nanocomposites was measured at room temperature based on the four-probe method (Figure 1) using specimens of dimensions $64 \times 13 \times 3 \mathrm{~mm}$ on a resistivity/Hall measurement system (Sony Tektronix 370 A, Japan). For this work, sharp diamond indenter was used and Vickers hardness was calculated by $1.854(\mathrm{~F} / \mathrm{D} 2)$, where $\mathrm{F}$ and $\mathrm{D} 2$ are applied force and area of indentation respectively. Fracture toughness of neat and nanofilled epoxy was determined from static three-point tests of the single edge notch specimens. Each of these specimens was cycled 100 times between 4 and $40 \%$ of the peak load at $1 \mathrm{~Hz}$ and then statically tested. During the static tests, the change in specimen length was measured by recording the ram positions through the displacement transducer of the universal tensile testing machine (Acumen eletrodynamic test system, MTS, USA). At least 5 samples (dimension: $64 \times 13 \times 3 \mathrm{~mm}$ ) were tested for evaluating fracture toughness. The critical stress intensity factor $\left(K_{\mathrm{IC}}\right)$ was calculated according to the Equations (1) and (2) [21]:

$$
K_{\mathrm{IC}}=\left(\frac{P}{B} w^{3 / 2}\right) f(a / w)
$$

where $P$ applied load on the specimen, $B$ specimen thickness, $w$ specimen width, $a$ crack length, and
Fractured surfaces were gold coated after indentation and observed in an FE-SEM (FEI, Inspect F, $20 \mathrm{kV}$, USA). For measuring the percolation threshold, circular discs of about $0.8 \mathrm{~mm}$ thickness were cut and the cross-sectional areas were coated with conductive silver paint. Prior to silver coating, all sample surfaces were cleaned by gentle wiping using a lint-free cloth soaked with isopropanol. The diameters of circles with equivalent area to the crosssection were much greater than the distance between them. 2-probe AC impedance spectroscopy was performed using a Solartron 1260 Impedance/gain phase analyser with a voltage amplitude of $1 \mathrm{~V}$. The frequency range was set from 1 to $107 \mathrm{~Hz}$. All electrical conductivities were measured at $42 \%$ humidity.

\section{Results and discussion}

Structural health assessing for nanostructured carbon-filled epoxy composites was verified by measuring the change in electrical resistivity after systematic introduction of damage to the samples. The experimental setup shown in Figure 1 was especially designed for the evaluation of the change in electrical conductivity during the incremental damage. It should be noted that the connecting wires in the experimental setup (Figure 1) were permanently bonded by using silver paste in order to avoid any contact resistance for this comparative analysis. Following Vickers indentation, clear damage was visible to the unaided eye for all specimens.

During Vickers indentation, brittle materials usually fracture under the surface or sub-surface (Hertzian cone crack) and from the tips of the diagonal indent to accommodate the penetrating diamond indenter [22]. As a result of that, new surfaces are created and radial-median and lateral crack systems are produced during loading and unloading of Vickers indenter (Figure 2). Electronic microscopy revealed radial cracking from the tip of the indent (Figure 3). All samples (epoxy, epoxy -0.2 vol\% CB and epoxy $0.2 \mathrm{vol} \% \mathrm{CNT}$ nanocomposites) retained their integrity because of the small Vickers loadings after indenta-

$$
f(a / w)=\frac{\sqrt[3]{\frac{a}{w}}\left[1.99-\left(\frac{a}{w}\right)\left(1-\frac{a}{w}\right)\left(2.15+3.93 \frac{a}{w}+2.7 \frac{a^{2}}{w^{2}}\right)\right]}{2\left(1+2 \frac{a}{w}\right)\left(1-\frac{a}{w}\right)}
$$




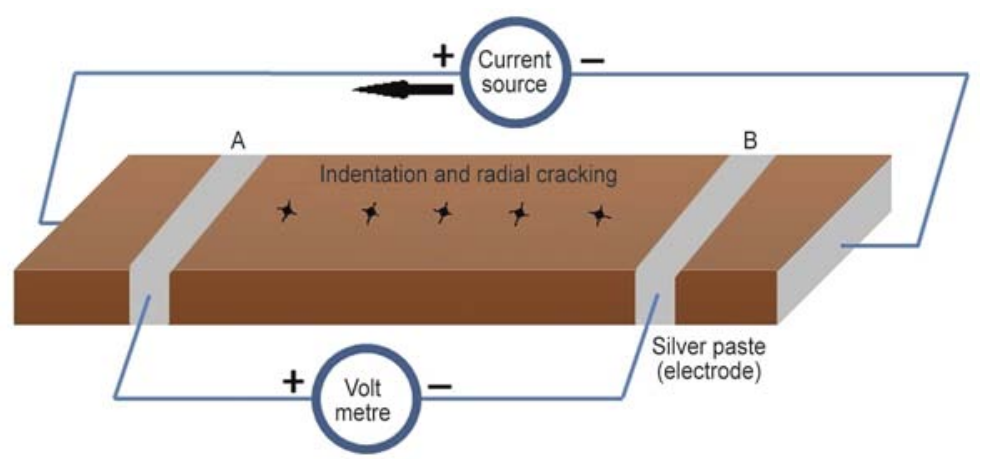

Figure 1. Four-probe electrical conductivity measurement schematics of indented sample. Distance between A and B is $56 \mathrm{~mm}$
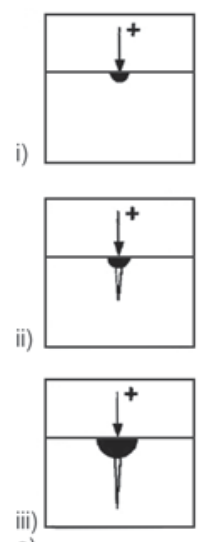

a)

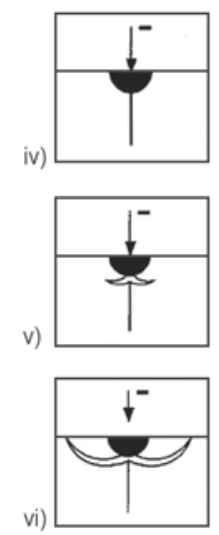

Figure 2. Radial-median and lateral crack system: a) evolution during complete loading $(+)$ and unloading $(-)$ cycle. Dark region denotes irreversible deformation zone; and b) geometrical parameters of radial system [22].

tion. The load $(500 \mathrm{~g})$ was carefully selected to cause appropriate sub-surface damage for analysing electrical conductivities without completely fracturing the sample bars.

As compared to epoxy, with a Vickers hardness of $0.16 \pm 0.02 \mathrm{GPa}$, slight decreases in hardness were observed for epoxy -0.2 vol\% CB and epoxy 0.2 vol $\%$ CNT nanocomposite samples (i.e. $0.12 \pm 0.03$ and $0.11 \pm 0.03 \mathrm{GPa}$ respectively). This is primarily due to the lubricant nature of carbon nanofillers which is responsible for deeper penetration on the Vickers indenter during the indentation. These observations for nanocomposites and measured values of hardness for epoxy are in consistent with study conducted by Low and Shi [23] and Lau et al. [2] respectively. Due to fibrous nature of CNTs, crack bridging was observed (Figure 4) which is also responsible for improving fracture toughness of epoxy nanocomposites [24]. Such phenomenon was not observed for particulate (carbon black) filled epoxy nanocomposites where isolated $\mathrm{CB}$ particles were observed on

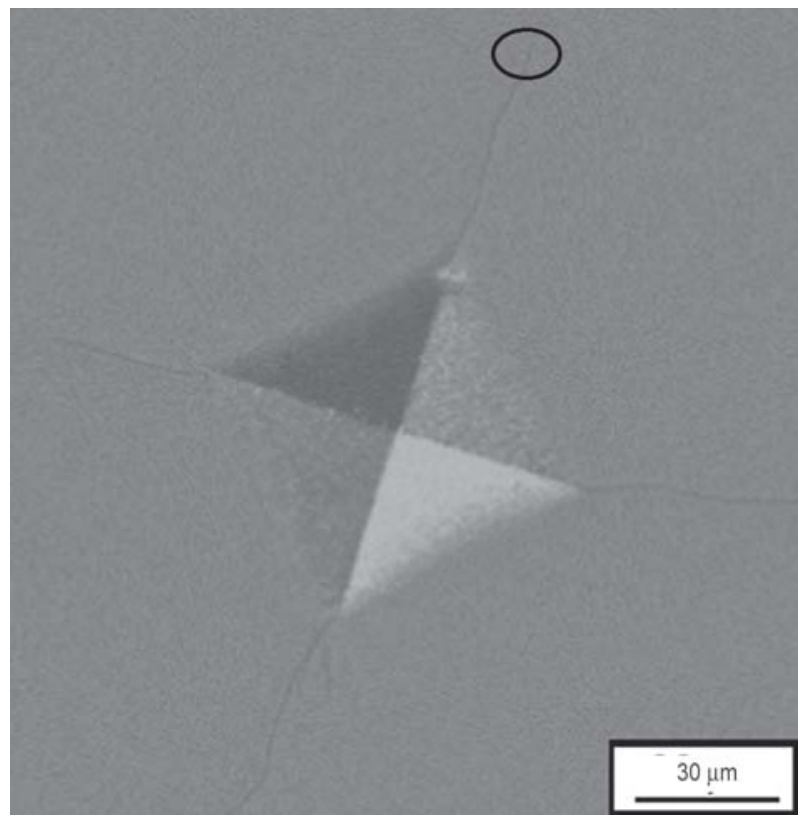

Figure 3. Vickers indent on epoxy -0.2 vol\% CB nanocomposite showing radial cracking. Tip of the crack (encircled) is analysed in Figure 4.

the edges of the fractured surfaces (Figure 4). This observation is also consistent with previous research work comparing mechanical properties of CNT and CB-filled epoxy nanocomposites [3].

In order to efficiently exploit the full potential of CNTs for improving the sensing capability in polymers, it is important to have good dispersion of CNT in the polymeric matrix. The extraordinary large specific surface area of CNTs is the main hurdle for homogeneous dispersion and de-bundling of CNTs. Calendering epoxy-nanofiller dispersions has proved to be the one of the best available solution for producing homogeneous epoxy-nano filler dispersions [3]. Individual CB particles, pointed by white arrows, and CNT fibres can be seen in Figure $4 a$ and $4 b$ respectively. Particularly for epoxy -0.2 vol $\%$ CNT nanocomposites, this level of dispersion is critical 


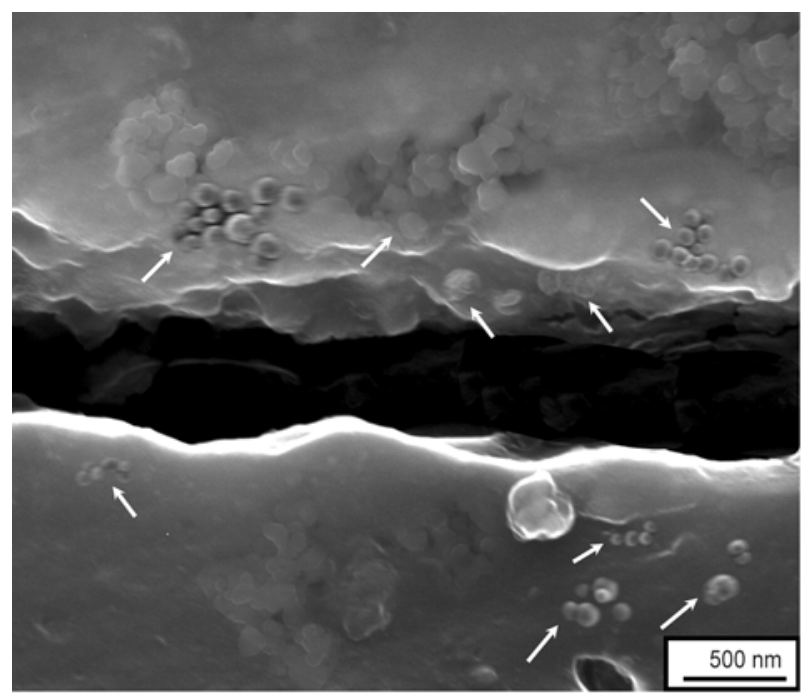

a)

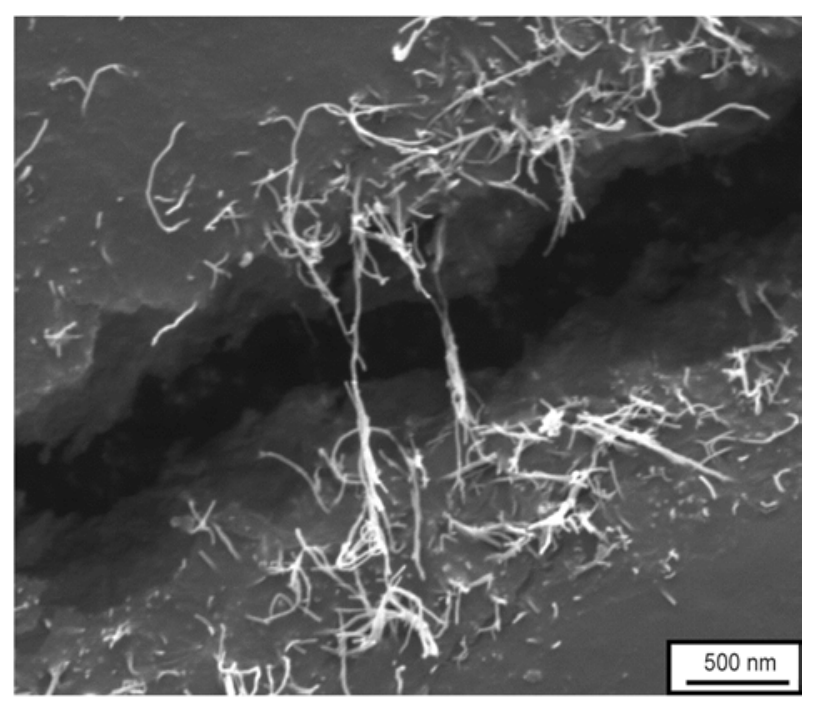

b)

Figure 4. Damaged caused by Vickers indentation in: a) epoxy -0.2 vol $\%$ CB nanocomposite; and b) epoxy -0.2 vol $\%$ CNT nanocomposite

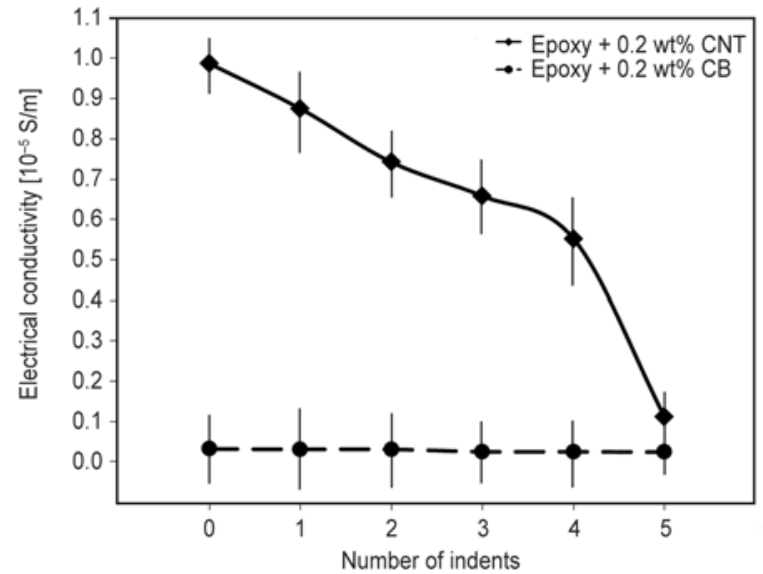

Figure 5. Change in electrical conductivity due to Vickers indentation in epoxy - carbon nanocomposites

for its improved damage sensing capability. It can be understood that if CNTs are not homogeneously distributed and there is no separation of CNTs taking place during the fracturing, such high sensitivity (Figure 5) cannot be achieved.

It is necessary to discuss the fracture, hardness and location of carbon nanofillers to understand the induced structural health assessing capability, which can be observed from Figure 5. The change in electrical conductivities of epoxy $-0.2 \mathrm{vol} \% \mathrm{CB}$ and epoxy -0.2 vol\% CNT nanocomposite samples as a result of the deliberate damage (i.e. Vickers indentation) is shown in Figure 5. Electrical conductivity was measured after every indentation for all 5 samples of both types of nanocomposites. Error bars, shown in Figure 5, represent good repeatability of measured values. It is obvious from Figure 5 that
CNT-filled epoxy nanocomposites were more sensitive towards the indentation damage as compared to CB-filled epoxy nanocomposites. After 5 indentations, the average electrical conductivity for CNT nanocomposites decreased from $0.987 \cdot 10^{-5}$ to $0.111 \cdot 10^{-5} \mathrm{~S} / \mathrm{m}$ (i.e. a decrease of $89 \%$ ). For CB nanocomposites, the average electrical conductivity decreased from $0.032 \cdot 10^{-5}$ to $0.024 \cdot 10^{-5} \mathrm{~S} / \mathrm{m}$ (i.e. a decrease of $25 \%$ ). This is due to the fibrous nature of CNTs as opposed to particulate form of CB (Figure 4). The different behaviour of the carbon nanotube modified matrix system and the carbon black modified matrix system has to be attributed to the intrinsic structure of the percolated conductive paths in the composite. In this context, the high axial electrical conductivity CNT not only offers the potential for fabricating conducting polymers but also responsive polymeric systems.

For correlating change in electrical conductivity and indentation damage, fracture toughness of samples was compared (Figure 6). It was found that CNT nanocomposites have 14\% higher fracture toughness as compared to $\mathrm{CB}$ nanocomposites. This means that upon indentation, more damage was done by the penetrating diamond indenter for CB nanocomposites as compared to CNT nanocomposites. However, very small change in electrical conductivity was observed for CB nanocomposites. For CNT nanocomposites, CNTs are responsible for improving fracture toughness of epoxy matrix, which is also consistent with the literature [3, 9]. As compared to $\mathrm{CB}$ nanocomposites, less damage was produced in 


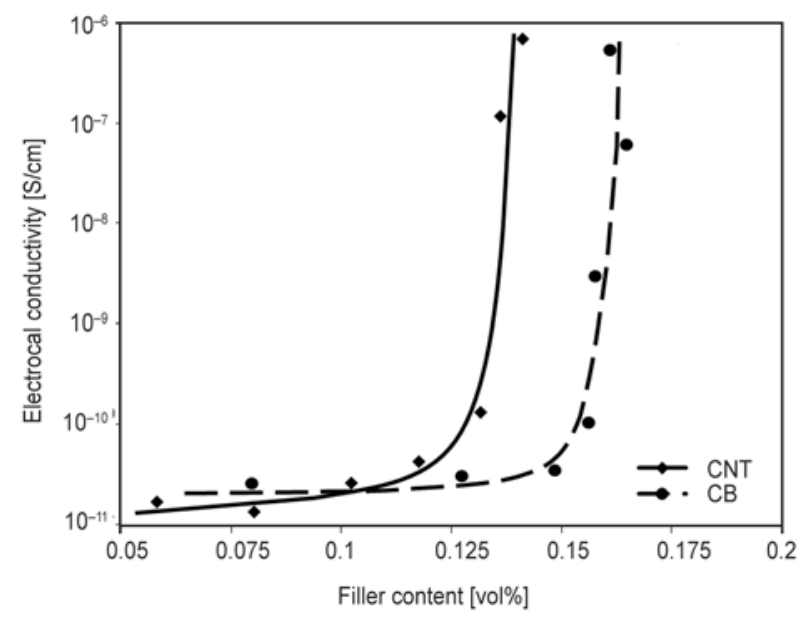

Figure 6. Epoxy nanocomposite conductivity as a function of carbon nanofiller volume fraction

CNT nanocomposites but a sharp decrease in electrical conductivity was observed. This means that CNTs nanocomposite have superior damage sensing capability as compared to $\mathrm{CB}$ nanocomposites, which is due to the fibrous nature and high surface area of CNTs.

Both carbon fillers used in this study have different geometrical shapes (i.e. CNTs are cylindrical, whereas $\mathrm{CB}$ consists of spherical particles). For the sake of real and comprehensive comparison, it is necessary to evaluate the percolation threshold for epoxy nanocomposites. Percolation thresholds for epoxy - carbon nanocomposites were measured (Figure 7). A percolation threshold of 0.0129 and 0.158 vol\% was found for epoxy - CNT and epoxy - CB nanocomposites respectively. Therefore, it is confirmed that by using $0.2 \mathrm{vol} \%$ of filler content for comparing structural health assessing capabilities, the authors are making sure that the filler content is

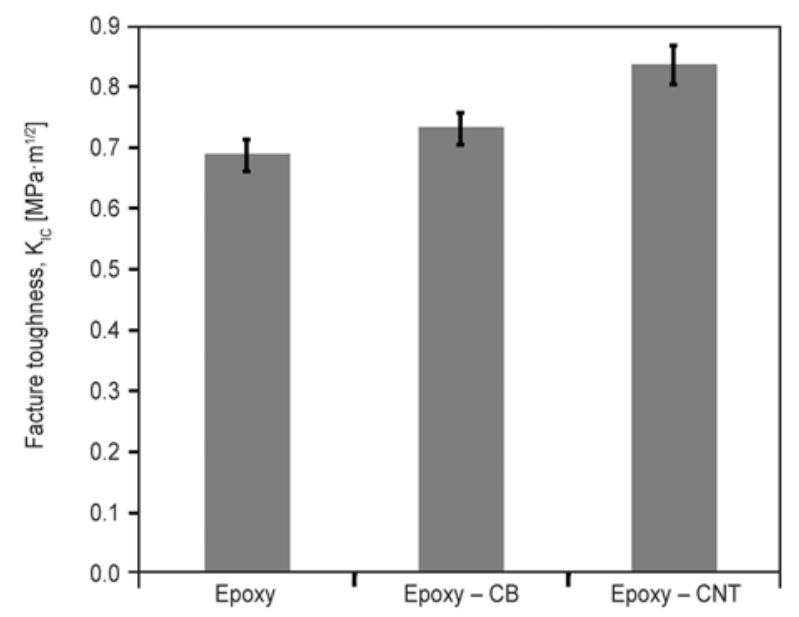

Figure 7. Fracture toughness of neat epoxy and epoxy nanocomposites more than the percolation threshold which is important for this work. These percolation threshold and electrical conductivity values for epoxy nanocomposites are consistent with the previously reported values as reviewed by Bauhofer and Kovacs [25].

\section{Conclusions}

The results of the experiments presented here demonstrate the high potential of CNTs to be used for damage sensing in brittle materials like epoxy and glasses. CNTs were used to sense sub-surface damage in cured epoxy. All nanocomposite compositions used in this study had filler content higher than the percolation threshold for electrical conductivity. As compared to $\mathrm{CB}, \mathrm{CNTs}$ possess better damage sensing ability in brittle nanocomposite structures due to their high aspect ratio (fibrous nature) and electrical conductivity. It was found that CNT nanocomposites have $14 \%$ higher fracture toughness as compared to $\mathrm{CB}$ nanocomposites. A sharp decrease of $89 \%$ was observed in the electrical conductivity of epoxy - CNT nanocomposite as compared to $25 \%$ in the electrical conductivity of epoxy - CB nanocomposite due to indentation damage. Therefore, it is concluded that as compared to CB, CNT offer higher sensitivity for structural health assessing to diagnose a structural safety and to prevent a catastrophic failure in brittle materials. There are many challenges for practical implementation of proposed novel NDE technique for a real structural unit and it would be the subject of the future research. This technique can also be used for damage sensing in ceramics matrices, which is subject of future research.

\section{Acknowledgements}

Dr. Fawad Inam would like to acknowledge generous support from Airbus UK, Cytec Engineered Materials Ltd. and Thomas Swan for supplying materials and their valuable time.

\section{References}

[1] Iijima S.: Helical microtubules of graphitic carbon. Nature, 354, 56-58 (1991).

DOI: $10.1038 / 354056 a 0$

[2] Lau K-T., Shi S-Q., Zhou L-M., Cheng H-M.: Microhardness and flexural properties of randomly-oriented carbon nanotube composites. Journal of Composite Materials, 37, 365-376 (2003).

DOI: $10.1177 / 0021998303037004043$ 
[3] Gojny F. H., Wichmann M. H. G., Köpke U., Fiedler B., Schulte K.: Carbon nanotube-reinforced epoxycomposites: Enhanced stiffness and fracture toughness at low nanotube content. Composites Science and Technology, 64, 2363-2371 (2004).

DOI: $10.1016 /$ j.compscitech.2004.04.002

[4] Inam F., Peijs T.: Transmission light microscopy of carbon nanotubes-epoxy nanocomposites involving different dispersion methods. Advanced Composites Letters, 15, 7-13 (2006).

[5] Prashantha K., Soulestin J., Lacrampe M. F., Krawczak P.: Present status and key challenges of carbon nanotubes reinforced polyolefins: A review on nanocomposites manufacturing and performance issues. Polymers and Polymer Composites, 17, 205-245 (2009).

[6] Cho J. C., Inam F., Reece M. J., Chlup Z., Dlouhy I., Shaffer M. S. P., Boccaccini A. R.: Carbon nanotubes: Do they toughen brittle matrices? Journal of Materials Science, 46, 4770-4779 (2011).

DOI: $10.1007 / \mathrm{s} 10853-011-5387-\mathrm{X}$

[7] Bakshi S. R., Lahiri D., Agarwal A.: Carbon nanotube reinforced metal matrix composites - A review. International Materials Reviews, 55, 41-64 (2010). DOI: $10.1179 / 095066009 X 12572530170543$

[8] De Volder M. F. L., Tawfick S. H., Baughman R. H., Hart A. J.: Carbon nanotubes: Present and future commercial applications. Science, 339, 535-539 (2013). DOI: $10.1126 /$ science.1222453

[9] Zhou Y. X., Wu P. X., Cheng Z-Y., Ingram J., Jeelani S.: Improvement in electrical, thermal and mechanical properties of epoxy by filling carbon nanotube. Express Polymer Letters, 2, 40-48 (2008).

DOI: $10.3144 /$ expresspolymlett.2008.6

[10] Lee S-I., Yoon D-J.: Structural health monitoring for carbon fiber/carbon nanotube (CNT)/epoxy composite sensor. Key Engineering Materials, 321-323, 290-293 (2006).

DOI: 10.4028/www.scientific.net/KEM.321-323.290

[11] Böger L., Wichmann M. H. G., Meyer L. O., Schulte K.: Load and health monitoring in glass fibre reinforced composites with an electrically conductive nanocomposite epoxy matrix. Composites Science and Technology, 68, 1886-1894 (2008).

DOI: 10.1016/j.compscitech.2008.01.001

[12] Alexopoulos N. D., Bartholome C., Poulin P., MarioliRiga Z.: Structural health monitoring of glass fiber reinforced composites using embedded carbon nanotube (CNT) fibers. Composites Science and Technology, 70, 260-271 (2010).

DOI: 10.1016/j.compscitech.2009.10.017

[13] Anand S. V., Mahapatra D. R.: Quasi-static and dynamic strain sensing using carbon nanotube/epoxy nanocomposite thin films. Smart Materials and Structures, 18, 045013/1-045013/13 (2009).

DOI: $10.1088 / 0964-1726 / 18 / 4 / 045013$
[14] Thostenson E. T., Chou T-W.: Carbon nanotube-based health monitoring of mechanically fastened composite joints. Composites Science and Technology, 68, 18861894 (2008). DOI: $10.1016 /$ j.compscitech.2008.05.016

[15] Naghashpour A., Hoa S. V.: In situ monitoring of through-thickness strain in glass fiber/epoxy composite laminates using carbon nanotube sensors. Composites Science and Technology, 78, 41-47 (2013).

DOI: 10.1016/j.compscitech.2013.01.017

[16] Kostopoulos V., Vavouliotis A., Karapappas P., Tsotra P., Paipetis A.: Damage monitoring of carbon fiber reinforced laminates using resistance measurements. Improving sensitivity using carbon nanotube doped epoxy matrix system. Journal of Intelligent Material Systems and Structures, 20, 1025-1034 (2009). DOI: $10.1177 / 1045389 X 08099993$

[17] Montalvão D., Maia N. M. M., Ribeiro A. M. R.: A review of vibration-based structural health monitoring with special emphasis on composite materials. The Shock and Vibration Digest, 38, 295-324 (2006). DOI: $10.1177 / 0583102406065898$

[18] Ciang C. C., Lee J-R., Bang H-J.: Structural health monitoring for a wind turbine system: A review of damage detection methods. Nanotechnology, 22, 045017/1045017/5 (2013).

DOI: $10.1088 / 0957-0233 / 19 / 12 / 122001$

[19] Yu X., Kwon E.: A carbon nanotube/cement composite with piezoresistive properties. Smart Materials and Structures, 18, 055010/1-055010/5 (2009).

DOI: $10.1088 / 0964-1726 / 18 / 5 / 055010$

[20] Inam F., Vo T., Jones J. P., Lee X.: Effect of carbon nanotube lengths on the mechanical properties of epoxy resin: An experimental study. Journal of Composite Materials, 47, 2321-2330 (2013). DOI: $10.1177 / 0021998312457198$

[21] ASTM D5045-99(2007)e1: Standard test methods for plane-strain fracture toughness and strain energy release rate of plastic materials (2007).

[22] Lawn B.: Facture of brittle solids. Cambridge University Press (1993).

[23] Low I. M., Shi C.: Vickers indentation responses of epoxy polymers. Journal of Materials Science Letters, 19, 1181-1183 (1998). DOI: 10.1023/A:1006517005082

[24] Hollertz R., Chatterjee S., Gutmann H., Geiger T., Nüesch F. A., Chu B. T. T.: Improvement of toughness and electrical properties of epoxy composites with carbon nanotubes prepared by industrially relevant processes. Nanotechnology, 22, 125702/1-125702/9 (2011).

DOI: $10.1088 / 0957-4484 / 22 / 12 / 125702$

[25] Bauhofer W., Kovacs J. Z.: A review and analysis of electrical percolation in carbon nanotube polymer composites. Composites Science and Technology, 69, 1486-1498 (2009).

DOI: $10.1016 /$ j.compscitech.2008.06.018 